\title{
Explicações no ensino de ciências: revisando o conceito a partir de três distinções básicas
}

\author{
Explanations in science teaching: reviewing the concept from \\ three basic perspectives
}

Renato Felix Rodrigues ${ }^{1}$. Alexsandro Pereira de Pereira ${ }^{1}$

\begin{abstract}
Resumo: Explicar é uma das práticas discursivas mais importantes da ciência e do seu ensino. Nas últimas décadas, este tema passou a receber atenção de legislações educacionais, professores e pesquisadores, embora pesquisas sobre o uso de explicações no ensino de ciências destaquem a dificuldade de definir este conceito. Neste trabalho, revisamos o conceito explicação a partir de três distinções básicas: explicações e narrativas; explicações e argumentos; explicações científicas e explicações escolares. Este trabalho busca primeiramente trazer clareza para os conceitos aqui discutidos, enfatizando que explicações, argumentos e narrativas são elementos importantes, porém distintos da comunicação da sala de aula. Limites para a aproximação entre práticas explicativas com argumentos e narrativas são discutidos. O trabalho aponta também a importância do tema para o ensino de ciências e o baixo número de trabalhos dedicados a esta questão entre pesquisadores brasileiros.
\end{abstract}

Palavras-chave: Ensino de ciências. Explicações. Argumentos. Narrativas. Revisão da literatura.

\begin{abstract}
Explaining is among the most important discursive practice for science and its teaching. In the last decades this topic received attention from official documents, teachers and researchers. However, research in science teaching highlights the difficulty in defining this concept. In this paper we review the concept of explanation from three basic perspectives: explanations and narratives; explanation and arguments; scientific explanation and school science explanation. This work seeks firstly to bring clarity to the above-mentioned concepts, highlighting the fact that explanation, arguments and narratives are important but distinct elements of science classroom discourse. Limits in explanatory practices in relation to arguments and narratives are discussed. It also shows the importance of the topic for science teaching and the lack of research on explanations in Brazilian literature.
\end{abstract}

Keywords: Science teaching. Explanations. Arguments. Narratives. Literature review.

\footnotetext{
${ }^{1}$ Universidade Federal do Rio Grande do Sul (UFRGS), Instituto de Física, Porto Alegre, RS, Brasil. Orcid:

<https://orcid.org/0000-0003-2149-7995>. E-mail: <renato.felix@ufrgs.br>.
} 


\section{Introdução}

Explicar é uma das práticas discursivas mais importantes do ensino de ciências, e praticamente todo professor de ciências concorda que explicar é parte fundamental de seu trabalho. Professores explicam fenômenos (e.g., efeito estufa, movimento da Lua ao redor da Terra, observações experimentais), conceitos abstratos (e.g., equilíbrio químico, conservação de energia) e até coisas que parecem não precisar de explicação (e.g., porque o céu é escuro à noite ou porque humanos possuem pulmões) (OGBORN et al., 1996). Explicar é uma prática epistêmica autêntica da ciência, além de ser essencial para proporcionar a compreensão de ideias científicas (CHINN; MALHOTRA, 2002). Além disso, legislações educacionais têm passado a enfatizar o papel das explicações científicas no ensino de ciências, tanto no Brasil quanto no Exterior (e.g. BRASIL, 2002, 2015; NATIONAL RESEARCH COUNCIL, 2007).

O debate sobre a natureza da explicação científica tem uma longa história na filosofia da ciência. A origem dessa discussão é frequentemente atribuída ao trabalho seminal de Hempel e Oppenheim (1948) intitulado Studies in the logic of explanation. Neste trabalho é introduzida uma abordagem lógica às explicações científicas, dando origem a uma tradição de pesquisa que se tornou uma subárea da filosofia da ciência. Na educação em ciências, é a partir da década de 1990 que explicações passam a receber atenção de pesquisadores, inicialmente na literatura internacional (e.g. DAGHER; COSSMAN, 1992; GILBERT; BOULTER; RUTHERFORD, 1998a, 1998b; OGBORN et al., 1996) e posteriormente na literatura nacional (e.g., CUSTÓDIO; CRUZ; PIETROCOLA, 2011; MARTINS; OGBORN; KRESS, 1999; MORTIMER; SCOTT, 2002).

Apesar do aumento recente no número de artigos publicados sobre explicações, é possível observar uma falta de clareza sobre o significado da palavra explicação (OSBORNE; PATTERSON, 2011). Nas palavras de Norris et al. (2005, p. 545, tradução nossa), "é justo dizer que não possuímos uma explicação completamente satisfatória de explicação”. De modo similar, autores como Braaten e Windschitl (2011, p. 641, tradução nossa) argumentam que "existem muitas maneiras de conceitualizar explicações científicas, das quais todas podem ser relevantes para a pesquisa e prática na educação em ciências". Como resultado, as diferentes perspectivas sobre o que constitui uma explicação têm gerado certa ambiguidade conceitual nesse campo de estudo.

Com o objetivo de apresentar o panorama conceitual dentro do qual a noção de explicação tem sido discutida na educação em ciências, identificamos três distinções básicas: entre explicações e narrativas, entre explicações e argumentos e entre explicações científicas e explicações escolares. Este método de revisão da literatura é inspirado no trabalho de Wertsch e Roediger (2008), no qual os autores propõem três oposições básicas para revisar o conceito de memória coletiva nas ciências sociais e humanas. É importante salientar que autores como Mortimer e Scott (2002) já propuseram uma distinção entre descrição, explicação e generalização para especificar características básicas da linguagem social da sala de aula de ciências. No entanto, acreditamos que as distinções aqui propostas podem contribuir para colocar em evidência importantes controvérsias existentes na literatura sobre explicações ${ }^{2}$.

\footnotetext{
${ }^{2}$ Braaten e Windschitl (2011) também apresentam categorias para classificar o modo como a literatura em educação em ciências tem utilizado o termo explicação. Apesar de apontarem criticamente uma sobreposição entre explicações e argumentos, o objetivo dos autores não é distinguir explicações de outros gêneros comunicativos.
} 


\section{Escopo}

A aquisição dos trabalhos incluídos nesta revisão começou através da plataforma eletrônica Institute of Education Sciences (ERIC) ${ }^{3}$, procurando por artigos contendo o termo scientific explanation no título. Nessa primeira busca foram encontrados trinta e nove artigos. A essa amostra inicial adicionamos trabalhos encontrados através da busca pelo termo explicação científica na plataforma Google Acadêmico ${ }^{4}$. O termo explicação é utilizado em diversas situações, mas identificamos apenas três artigos na literatura nacional que problematizam seu significado no ensino de ciências. Durante a leitura desses trabalhos foram identificadas controvérsias relacionadas à definição de explicação. Tais controvérsias direcionaram nosso foco para trabalhos voltados para discussões desta natureza, servindo de base para a construção das categorias deste trabalho. Os artigos encontrados indicavam outros trabalhos influentes sobre este tema que haviam ficado de fora de nosso escopo inicial. Sua leitura nos levou a outras referências relevantes que, por sua vez, nos levaram a outras referências, e assim sucessivamente até chegarmos à lista final de referências incluídas aqui. Obviamente, essa forma de categorização não esgota o universo de perspectivas teóricas sobre explicação encontradas na literatura.

\section{Diferentes concepções de explicação na educação em ciências}

A falta de consenso no campo das pesquisas sobre explicação reflete na variedade de significados atribuídos a este termo. Martin (1972), por exemplo, sugere os seguintes significados: uma clarificação do significado de uma frase em contexto científico; uma justificação de uma crença ou ação; uma descrição causal para um estado, evento ou processo; uma citação de uma teoria da qual uma lei pode ser deduzida; uma atribuição de função a um objeto. Já Norris et al. (2005) abordam explicações tanto a partir do tipo quanto da função que desempenham. Com relação ao último, os autores dão ênfase às seguintes funções: expandir o significado, justificar, descrevere atribuir causa. Com relação ao primeiro, os autores destacam a explicação científica como sendo o tipo mais debatido na literatura, apresentando quatro padrões de explicação propostos por Nagel (1961): dedutivo, probabilistico, funcional e genético. Gilbert, Boulter e Rutherford (1998a), por sua vez, propõem uma tipologia de explicações a partir de respostas a perguntas sobre fenômenos naturais. De acordo com a classificação dos autores, uma explicação pode ser: intencional, descritiva, interpretativa, causal e preditiva. Finalmente, Braaten e Windschitl (2011) classificam o modo como o termo explicação é abordado na literatura em educação em ciências, identificando três formas básicas: explicação como explanaşão, explicação como causação e explicação como justificação.

Conforme mencionado na introdução deste artigo, em vez de buscar uma definição única para o conceito explicação, exploramos neste trabalho três distinções que retratam as principais controvérsias encontradas na literatura: entre explicaçōes e narrativas, entre explicaçōes e argumentos e entre explicações científicas e explicações escolares. A última distinção (entre explicações

\footnotetext{
${ }^{3}$ Disponível em: <https://eric.ed.gov/>. Acesso em: 22 jan. 2018.

${ }^{4}$ Disponível em: < https://scholar.google.com.br/>. Acesso em: 22 jan. 2018.
} 
científicas e explicações escolares) pode ajudar a esclarecer certas confusões conceituais que surgem nas discussões da primeira e da segunda distinção.

\section{Explicações e narrativas}

Uma primeira distinção a ser discutida neste trabalho é entre explicação e narrativa. Assim como ocorre com explicação, o significado de narrativa é notoriamente contestado. Seu estudo não se restringe apenas ao campo literário, sendo discutido também em áreas como antropologia, história e psicologia (RIBEIRO; MARTINS, 2007). Uma forma geral de definir este conceito é considerar narrativas como o ato verbal de contar a alguém que algo aconteceu (SMITH, 1981). Com base em ideias apresentadas por Bruner (1990), Ribeiro e Martins (2007) destacam que narrativas apresentam uma sequência cronológica de eventos; podem abordar tanto fatos reais quanto imaginários; necessitam de algo que as justifiquem, não se produzindo narrativas sobre fatos e comportamentos ordinários e previsíveis.

Para analisar o papel de narrativas no ensino de ciências, Norris et al. (2005) fazem uma distinção entre explicações extrínsecas e explicações intrínsecas à ciência. Explicações extrínsecas são explicações que envolvem informações sobre a ciência e que estão fora do corpo de conhecimento científico (e.g., biografia de cientistas). Explicações intrínsecas, por sua vez, são explicações que dão conta de fenômenos naturais e fazem parte do corpo de conhecimento científico (e.g., queda dos corpos). Com respeito a explicações extrínsecas, autores como Milne (1998) destacam a importância das narrativas relativas à história da ciência para transmitir uma cultura científica, descrevendo e situando os processos de produção do conhecimento científico. Na visão de Ribeiro e Martins (2007), narrativas desempenham tanto uma função informativa quanto avaliativa, no sentido de informar fatos que aconteceram e avaliar eventuais significados dos eventos ali narrados.

Além da inclusão de narrativas por meio da história da ciência, alguns pesquisadores defendem o uso de narrativas para produzir explicações intrínsecas à ciência. Atraídos pelos benefícios educacionais que narrativas presumivelmente proporcionam, autores como Millar e Osborne (1998, p. 2013, tradução nossa) consideram que narrativas podem ser úteis para "comunicar ideias e torná-las coerentes, memorizáveis e significativas”. Eles defendem um maior uso de narrativas na educação em ciências, considerando-as uma das formas mais poderosas de comunicar ideias. $\mathrm{Na}$ visão desses autores, narrativas constituem uma importante ferramenta para a educação em ciências, destacando seu potencial para organização e apresentação dos conteúdos, para a memorização e a compreensão, e para o aumento do interesse dos alunos. De fato, uma das maneiras de transformar conhecimentos em sala de aula é convertê-los em narrativas, fazendo-as atuar como um "veículo para o conhecimento" (OGBORN et al., 1996; MARTINS; OGBORN; KRESS, 1999).

Entre os autores que investem no uso de histórias para comunicar ideias científicas destacamos Ogborn et al. (1996). Eles consideram explicações científicas como sendo análogas a estórias.

\footnotetext{
${ }^{5}$ Utilizamos o termo "estórias" como tradução de "stories", para se referir a histórias fictícias criadas por um narrador.
} 
Nessa perspectiva, protagonistas dessas estórias possuem poderes próprios de ação e interagem em sequências de eventos que produzem o fenômeno a ser explicado. Um exemplo disso são elétrons que fluem por um material condutor produzindo uma corrente elétrica que faz com que uma lâmpada acenda. Esta visão explora o potencial explicativo das narrativas para comunicar ideias, e, consequentemente, promove uma aproximação entre explicações e narrativas.

Do outro lado do debate estão autores que questionam a relevância das narrativas na educação em ciências. Mortimer (2000), por exemplo, não descarta a possibilidade de uma explicação ser apresentada na forma de narrativa, mas considera que a maior parte das explicações de sala de aula aparece na forma de afirmações que pertencem a um modo de pensar que Bruner (1990) definiu como "paradigmático". Diferentemente do modo de pensar narrativo, que está relacionado ao ato de contar estórias, o modo paradigmático apresenta causas e busca por referências verificáveis empiricamente. Norris et al. (2005), por sua vez, investigam a existência de explicações na forma de narrativas, o que os autores denominam "explicações narrativas". A partir de uma revisão da literatura, eles reúnem uma série de elementos que caracterizam uma narrativa: (1) sequência de eventos (event-token); (2) narrador; (3) apetite narrativo; (4) tempo passado; (5) estrutura; (6) agência (agency); (7) propósito; e (8) leitor. É importante destacar que os autores não consideram que esses elementos formem um conjunto de condições necessárias e suficientes para constituir uma narrativa, mas sim que sua presença aumenta o grau de narratividade de um texto.

Norris et al. (2005) utilizam os critérios listados acima para analisar duas explicações presentes em livros textos: uma sobre o modelo de partículas das reações químicas (MILLAR; OSBONE, 1998) e outra sobre o meteoro que causou a extinção dos dinossauros (WARD, 1997). Com relação ao primeiro texto, os autores argumentam que não há o foco em eventos específicos e particulares associados a narrativas. Eles também apontam que o texto é fechado e linear, sem criar pontos de ambiguidade que convidem o leitor a ver múltiplos futuros possíveis e que provoquem estado de curiosidade, suspense ou tensão. Devido à ausência de importantes elementos narrativos, os autores consideram que este texto não se trata de uma narrativa; seria mais adequado considerá-lo uma prosa descritiva, que apresenta o porquê de certos tipos de eventos ocorrerem. Já com respeito ao segundo texto, os autores consideram que o trecho em questão se adéqua a uma narrativa por apresentar boa parte dos elementos citados anteriormente. Ele nomeia eventos que ocorreram no passado, um após o outro, e há tanto um narrador que relaciona o leitor a uma sequência de eventos quanto um clima de suspense gerado acerca do desfecho da história. Os autores consideram que o texto também é explicativo além de narrativo. A história apresenta uma sequência através da qual um sistema passou de um estado inicial para um estado final, apresentando as causas para o fenômeno a ser explicado. O fenômeno explicado por esta narrativa (a extinção dos dinossauros) também é intrínseco à ciência, o que sugere a possibilidade de construir explicações narrativas que sejam parte do conhecimento científico.

Esta visão também encontra respaldo na filosofia da ciência. Nagel (1961) classifica explicações narrativas (ou genéticas) como um dos seus quatro padrões de explicação científica. De acordo com Nagel (1961, p. 25, tradução nossa), explicações genéticas correspondem a estórias que resultam no evento a ser explicado, de modo que "a tarefa das explicações genéticas é estabelecer a sequência dos principais eventos através dos quais algum sistema anterior se transforma em um posterior". Ao compararem explicações e narrativas, Norris et al. (2005) observam que uma narrativa busca por instigar no leitor a vontade de conhecer o desfecho de 
uma estória particular, enquanto que o principal objetivo de uma explicação na ciência é apresentar regularidades generalizáveis para um certo conjunto de situações. Dessa forma, narrativas desempenham um papel importante na explicação de eventos únicos (como a extinção dos dinossauros ou o Big Bang). Por outro lado, o papel de narrativas na explicação científica se torna limitado quando a ciência busca por generalidade e não por particularidades, o que é frequente.

\section{Explicações e argumentos}

A segunda distinção que abordamos neste trabalho é entre explicações e argumentos. $\mathrm{Na}$ filosofia, argumentos são fundamentais para o estudo da lógica e da justificação de hipóteses (e.g., TOULMIN, 1958). Mas, assim como no caso de explicações e de narrativas, a definição de argumento também não é consensual. Jiménez-Aleixandre e Erduran (2007) destacam três características da atividade argumentativa: argumentação como justificação, argumentação como persuasão e argumentação como controvérsia. A primeira delas está relacionada ao ato de justificar uma hipótese, através da apresentação de linhas de raciocínio, teorias e evidências empíricas que a suportem. A segunda característica se refere ao ato de convencer alguém, enquanto que a terceira diz respeito ao debate entre dois lados com posições divergentes sobre um mesmo tema.

Durante as últimas duas décadas o papel de argumentos na educação em ciências tem recebido atenção de pesquisadores (e.g., BELL; LINN, 2000; OSBORNE; ERDURAN; SIMON, 2004; OSBORNE; PATTERSON, 2011; SAMPSON; CLARK, 2008; SÁ; KASSEBOEHMER; QUEIROZ, 2014; SASSERON; CARVALHO, 2011; ZOHAR; NEMET, 2002). Entre as razões utilizadas para pesquisar sobre argumentação, destaca-se o seu potencial para valorizar e proporcionar o pensamento crítico, expandir a compreensão de conceitos (BELL; LINN, 2000; BERLAND; McNEILL, 2010; ZOHAR; NEMET, 2002) e proporcionar a estudantes melhor compreensão da atividade e da natureza da ciência (BELL; LINN, 2000; DRIVER; NEWTON; OSBORNE, 2000; MILLAR; OSBORNE, 1998). Dessa forma, a habilidade de compreender e produzir argumentos é considerada crucial para o letramento científico (KUHN, 1993; NORRIS; PHILLIPS, 2003; SIMON; ERDURAN; OSBORNE, 2006). Argumentos são vistos também como uma forma de destacar aspectos sociais da construção de significados na ciência e ajudar a desenvolver critérios epistêmicos para avaliar conhecimentos (KNIGHT-BARDSLEY; McNEILL, 2016; OSBORNE; ERDURAN; SIMON, 2004; SIMON; ERDURAN; OSBORNE, 2006).

$\mathrm{Na}$ filosofia da ciência, Hempel e Oppenheim (1948) conceberam explicações científicas como argumentos dedutivos nos quais a conclusão afirma que o evento a ser explicado ocorre e as premissas justificam a ocorrência da conclusão ${ }^{6}$. Assim como ocorre na filosofia, há autores na Educação em Ciências que exploram a proximidade entre explicações e argumentos. Um exemplo de atividade identificada com frequência nessa literatura consiste em solicitar que estudantes produzam explicações sobre algum fenômeno observado e, em seguida, colocar as explicações produzidas em oposição umas com as outras (e.g., HOGAN; COREY, 2001; MCNEILL; LIZOTTE; KRAJCIK, 2006; SANDOVAL, 2003). Essa prática destaca a proximidade

\footnotetext{
${ }^{6}$ De forma semelhante, Kitcher (1989) considera que toda explicação é um argumento, embora nem todo argumento seja uma explicação. Tal afirmação deve ser entendida na perspectiva da lógica formal, segundo a qual argumentos são apresentados através de premissas e conclusões estruturadas de forma dedutiva ou indutiva.
} 
entre explicações e argumentos, estimulando os estudantes a convencer uns aos outros de que sua explicação é a mais adequada. Autores como Sandoval (2003) tratam explicações e argumentos como constituintes indissociáveis do discurso científico. Com esse respeito, Berland e Reiser (2009) defendem que explicação e argumentação são práticas científicas complementares e justificam que muitos pesquisadores em ensino de ciências as tratem como uma prática única, por elas serem interligadas epistemicamente e constituírem um gênero comunicativo específico.

Essa sobreposição entre explicações e argumentos que existe no ensino de ciências é bem ilustrada pela ferramenta instrucional desenvolvida por Katherine McNeill e seus colaboradores (MCNEILL, 2011; MCNEILL; KRAJCIK, 2008; MCNEILL; LIZOTTE; KRAJCIK, 2006). Tal ferramenta, uma das mais influentes na literatura, consiste de uma adaptação do modelo argumentativo de Toulmin (1958) e contém três componentes principais: hipótese (claim), a resposta a uma questão; evidência (evidence), informação ou dados que suportam a hipótese; e justificativa (reasoning), razão que mostra porque os dados contam como evidência para suportar a hipótese. Construir uma explicação nesta visão envolve gerar hipóteses sobre um fenômeno, conectar princípios científicos com a evidência disponível e justificar hipóteses usando evidências apropriadas. Nessa visão explicação e argumentação não são tratados separadamente, mas sim como uma prática única - à qual Berland e Reiser (2009) se referem como construção e defesa de explicaçôes cientificas.

Do outro lado do debate estão autores que consideram que associar a construção de explicações científicas com um objetivo persuasivo provoca falta de clareza sobre o que são explicações e o que são argumentos. Braaten e Windschitl (2011) chamam atenção para a dificuldade apresentada por educadores em ciências em distinguir explicação de argumentação quando tentam focar em uma dessas práticas científicas em particular. Abordar a atividade científica frequentemente implica em fazer um recorte segundo o qual alguma prática importante perde ênfase enquanto que outra recebe mais atenção. Esses autores consideram que trabalhos como os citados nos parágrafos anteriores promovem o foco na justificação de hipóteses utilizando evidências, mas que as hipóteses dos estudantes não são explicativas. Como resultado, tais atividades culminam em um predomínio de argumentação em detrimento de explicação, mesmo quando os pesquisadores alegam abordar explicações.

Na prática científica, explicações são julgadas por argumentos sobre o grau ao qual elas são coerentes, plausíveis e compreensíveis (THAGARD, 2014). Os dois, no entanto, não são a mesma coisa. Ao invés disso, são duas entidades discursivas: a explicação que tenta dar conta de um fenômeno dado e um argumento que examina a questão de a explicação ser válida ou não - isto é, se ela tem sucesso em produzir compreensão e se é melhor que tentativas concorrentes (OSBORNE; PATTERSON, 2011). Na filosofia da ciência, enquanto que um argumento mostra se um raciocínio é logicamente válido (no caso de um argumento dedutivo) ou provável (no caso de um argumento indutivo), uma explicação altera nossa percepção de um fenômeno ao proporcionar fatores que sejam explicativamente relevantes. Uma forma de distinguir explicações de argumentos é o fato de que as condições que tornam algo uma boa explicação diferem das condições para que seja um argumento válido. Brigandt (2016) articula este ponto em termos de explicações e argumentos buscarem padrões de adequação distintos, que decorrem de suas funções epistêmicas diversas. Enquanto que o objetivo de uma explicação é proporcionar compreensão sobre o porquê de um fenômeno ocorrer (ou porque um objeto possui certas características), o objetivo de um argumento é convencer alguém de que uma hipótese é verdade. 
Autores como Brigandt (2016) e Osborne e Patterson (2011) não se opõem nem discordam do importante papel que argumentos possuem tanto para a atividade científica como para o ensino de ciências. A questão destacada é o predomínio dado a atividades argumentativas às custas de atividades explicativas. É vital para o ensino de ciências ter clareza sobre o que distingue uma boa explicação de uma explicação ruim e os padrões que tornam um enunciado científico uma explicação, dado que nem todo argumento ou inferência científica a partir de evidência é uma explicação. Nesse sentido, Osborne e Patterson $(2011,2012)$ defendem que há um valor educacional em separar o ato de construir uma explicação e o ato de se engajar em um argumento. Segundo esses autores, uma vez que um estudante possa distinguir entre os atos epistêmicos de argumento e explicação, é mais fácil criar relações entre as atividades e transferir tal compreensão para um novo contexto. No entanto, será difícil para um estudante fazer tal distinção se (a) elas não estiverem claras em suas mentes e nas de seus professores e (b) se tais elementos não forem apontados explicitamente (OSBORNE; PATTERSON, 2011).

\section{Explicações científicas e explicações escolares}

A terceira distinção apresentada nesta revisão é entre explicações científicas e explicações escolares. As explicações científicas dizem respeito às explicações produzidas na atividade científica, divulgadas normalmente na forma de artigos científicos. Já as explicações escolares são explicações produzidas no contexto da sala de aula de ciências. Essa distinção foi originalmente proposta por Custódio, Cruz e Pietrocola (2011), embora o foco do trabalho desses autores tenha se concentrado na influência de fatores psicológico no entendimento de explicações escolares.

No que se refere às explicações científicas, os primeiros a estudar de forma sistemática sua estrutura lógica foram os filósofos da ciência. De acordo com o modelo pioneiro de Hempel e Oppenheim (1948), uma explicação sólida é dividida em dois componentes básicos: o explanandum e o explanans. O primeiro consiste de afirmações descrevendo o fenômeno a ser explicado, enquanto que o segundo especifica condições iniciais e ao menos uma lei geral. Atualmente, dois dos modelos mais aceitos pela filosofia da ciência são a abordagem Causal de Salmon (1984) e a abordagem de Unificação de Kitcher (1981). Segundo a perspectiva de Salmon, alcançamos compreensão sobre um fenômeno através do conhecimento de suas causas. Uma interação é considerada causal quando um sistema passa a apresentar uma nova característica que ele não apresentaria caso a interação em questão não houvesse ocorrido (SALMON, 1989). Já a teoria de Unificação considera que o entendimento científico é atingido quando uma explicação unifica fenômenos aparentemente desconexos. Segundo esta perspectiva, o poder explicativo das teorias científicas deriva de sua habilidade em empregar as mesmas leis gerais para explicar fenômenos diferentes através de contextos distintos (BRAATEN; WINDSCHITL, 2011).

Com relação às explicações escolares, diversos autores buscam caracterizar as formas de discurso explicativo que surgem nas aulas de ciências. Mortimer (2000), por exemplo, analisa a relação entre fenômenos macroscópicos e o mundo microscópico por meio da transição entre explicações empiricamente baseadas e explicações teoricamente orientadas. Nesse modelo, explicações que recorrem a entidades criadas dentro do discurso teórico da ciência são caracterizadas como teoricamente orientadas. Já explicações que recorrem a propriedades ou constituintes de um sistema que sejam diretamente observáveis são caracterizadas como empiricamente baseadas. Outro modelo é o quadro teórico de Martins, Ogborn e Kress (1999). Focando no discurso de 
professores de ciências, os autores identificam quatro momentos relacionados à construção de significados através de explicações: (i) criando diferenças, relacionada ao modo como professores apontam a necessidade de uma explicação em sala de aula; (ii) construindo entidades, que consiste na introdução de construtos teóricos que fazem parte do discurso científico; (iii) transformando conhecimentos, relacionada às mudanças que os professores fazem no conteúdo científico para torná-lo mais compreensível; e (iv) atribuindo significados ao que é material, referente ao uso de demonstrações experimentais para que alunos enxerguem o mundo a partir de uma perspectiva científica. Mais recentemente, Pereira, Lima Junior e Rodrigues (2016) abordaram as explicações como ferramentas culturais. Baseados na teoria sociocultural, os autores propuseram novas formas de caracterizar as explicações de sala de aula: (1) explicações como respostas dialógicas a outros enunciados; (2) explicações como instrumento de poder e autoridade nas relações sociais; (3) resistência como parte constitutiva do processo de domínio de explicações científicas.

Essa distinção entre explicações científicas e explicações escolares pode desfazer certas confusões conceituais existentes no estudo das explicações no ensino de ciências. Berland e McNeill (2012), por exemplo, apontam a importância de propiciar aos alunos a oportunidade de se engajar na construção de explicações. As autoras argumentam que é através do debate que explicações são criadas na atividade científica e que o objetivo do ensino de ciências não é apenas proporcionar a compreensão de conhecimentos científicos, mas também o conhecimento de práticas epistêmicas da ciência, como a construção de hipóteses verificáveis experimentalmente e a generalização de resultados particulares. Dessa forma, as autoras defendem a realização de atividades que simulem o fazer científico em sala de aula, na qual o gênero explicativo se sobrepõe a outras formas de discurso científico (e.g., argumentos, narrativas, etc.). Ao mesmo tempo, as autoras criticam a artificialidade de ensinar a estrutura lógica das explicações de forma explicita. Na visão das autoras, em práticas científicas reais essas diferentes formas de discurso são complementares e operam de forma sinergética.

Conforme alguns autores apontam, no entanto, as explicações atendem a objetivos distintos na atividade científica e no ensino de ciências. Enquanto que explicações na ciência buscam propor causas ou relações que justifiquem a ocorrência de fenômenos e criam novos conhecimentos, o ensino de ciências está preocupado em desenvolver compreensão sobre um corpo de conhecimento preexistente (CUSTÓDIO; CRUZ; PIETROCOLA, 2011). De acordo com Osborne e Patterson (2012), qualquer tipo de prática realizada com propósitos educacionais será apenas uma aproximação de práticas científicas autênticas. Isto significa que construir explicações em sala de aula e distinguir seus elementos deixa de ser uma atividade científica autêntica rapidamente, mas fazer tal julgamento seria um erro categórico já que a questão não é se esta é uma atividade científica autêntica, mas se esta é uma atividade educacional autêntica - um julgamento para o qual critérios distintos devem ser aplicados (OSBORNE; PATTERSON, 2012).

\section{Conclusão}

Aqui o termo explicação foi revisado a partir de trabalhos que discutem o significado deste conceito. Nas duas primeiras distinções explicações são contrastadas com argumentos e narrativas. Já a terceira distinção aborda trabalhos que discutem a relação entre explicações utilizadas na atividade científica e no ensino de ciências, apresentando diferentes posições 
acerca da proximidade entre as duas práticas e indicando características do ensino de ciências que podem limitar tal aproximação.

A aproximação entre explicações e narrativas na atividade científica é possível em casos restritos onde são apresentadas sequências de eventos através das quais algum sistema inicial se transformou em outro. Já quando a ciência busca por generalizações, e não por particularidades, o enunciado perde características importantes do gênero narrativo. $\mathrm{Na}$ atividade científica explicações e argumentos são consideradas práticas próximas, uma vez que as explicações propostas por cientistas precisam ser defendidas para que novas ideias possam ser aceitas pela comunidade científica. Já no ensino de ciências essa proximidade entre as práticas têm produzido opiniões divergentes quanto a tornar a distinção entre elas explícita para os alunos. Consideramos que no ensino de ciências é importante que professores tenham clareza sobre a natureza de distinções como essas, para que possam dar o suporte necessário para engajar seus estudantes em práticas explicativas e argumentativas adequadamente.

Com este trabalho buscamos alcançar a dois objetivos: o primeiro é o de contribuir para produzir clareza sobre os conceitos aqui discutidos, enfatizando que explicações, argumentos e narrativas são elementos importantes porém distintos da comunicação científica. O segundo objetivo é o de apontar a importância do tema para o ensino de ciências e o baixo número de trabalhos dedicados a esta questão entre os pesquisadores brasileiros. Este trabalho está longe de esgotar o debate sobre esta questão, mas acreditamos se tratar de um passo inicial no sentido de trazer mais clareza de linguagem e significado para o ensino de ciências.

\section{Agradecimentos}

Os autores agradecem à Coordenação de Aperfeiçoamento de Pessoal de Nível Superior (Capes) e ao Conselho Nacional de Desenvolvimento Científico e Tecnológico (CNPq), projeto $\mathrm{N}^{\circ} 441683 / 2014-2$, pelo financiamento recebido.

\section{Referências}

BELL, P.; LINN, M. C. Scientific arguments as learning artifacts: designing for learning from the web with KIE. International Journal of Science Education, Abingdon, v. 22, n. 8, p. $797-817,2000$.

BERLAND, L. K.; McNEILL, K. L. A learning progression for scientific argumentation: understanding student work and designing supportive instructional contexts. Science Education, Hoboken, v. 94, n. 5, p. 765-793, 2010.

For whom is argument and explanation a necessary distinction?: a response to Osborne and Patterson. Science Education, Hoboken, v. 96, n. 5, p. 808-813, 2012.

BERLAND, L. K.; REISER, B. J. Making sense of argumentation and explanation. Science Education, Hoboken, v. 93, n. 1, p. 26-55, 2009. 
Explicações no ensino de ciências: revisando o conceito ...

BRAATEN, M.; WINDSCHITL, M. Working toward a stronger conceptualization of scientific explanation for science education. Science Education, Hoboken, v. 95, n. 4, p. 639-669, 2011.

BRASIL. Ministério da Educação. Secretaria de Educação Básica. Base nacional curricular comum. Brasília, 2015.

BRASIL. Ministério da Educação. Secretaria da Educação Média e Tecnológica. Parâmetros curriculares nacionais $+(\mathbf{P C N}+)$ : ciências da natureza e suas tecnologias. Brasília, 2002.

BRIGANDT, I. Why the difference between explanation and argument matters to science education. Science \& Education, Dordrecht, v. 25, n. 1, p. 251-275, 2016.

BRUNER, J. Acts of meaning. Cambridge: Harvard University Press, 1990.

CHINN, C.; MALHOTRA, B. Epistemologically authentic inquiry in schools: a theoretical framework for evaluating inquiry tasks. Science Education, Hoboken, v. 86, n. 2, p. 175-218, 2002.

CUSTÓDIO, J. F.; CRUZ, F. F. S.; PIETROCOLA, M. Explicações científicas, explicações escolares e entendimento. Alexandria, Florianópolis, v. 4, n. 2, p. 179-204, 2011. Disponível em: <https://periodicos.ufsc.br/index.php/alexandria/article/view/37687/28858>. Acesso em: 22 jan. 2018.

DAGHER, Z; COSSMAN, G. Verbal explanations given by science teachers: their nature and implications. Journal of Research in Science Teaching, Hoboken, v. 29, n. 4, p. 361-374, 1992.

DRIVER, R.; NEWTON, P.; OSBORNE, J. Establishing the norms of scientific argumentation in classrooms. Science Education, Hoboken, v. 84, n. 3, p. 287-312, 2000.

GILBERT, J. K.; BOULTER, C. J.; RUTHERFORD, M. Models in explanations, part 1: horses of courses? International Journal of Science Education, Abingdon, v. 20, n. 1, p. 83-97, 1998a.

. Models in explanations, part 2: whose voice?: whose ears? International Journal of Science Education, Abingdon, v. 20, n. 2, p. 187-203, 1998 b.

HEMPEL, C. G.; OPPENHEIM, P. Studies in the logic of explanation. Philosophy of Science, Chicago, v. 15, n. 2, p. 135-173, 1948.

HOGAN, K.; COREY, C. Viewing classrooms as cultural contexts for fostering scientific literacy. Anthropology \& Education Quarterly, Arlington, v. 32, n. 2, p. 214-243, 2001.

JIMÉNEZ-ALEIXANDRE, M. P.; ERDURAN, S. Argumentation in science education: an overview. In: ERDURAN, S.; JIMÉNEZ-ALEIXANDRE, M. P. (Org.). Argumentation in science education: perspectives from classroom-based research. Dordrecht: Springer, 2007. p. 3-27.

KITCHER, P. Explanatory unification. Philosophy of Science, Chicago, v. 48, n. 4, p. 507-531, 1981. 
KITCHER, P. Explanatory unification and the causal structure of the world. In: KITCHER, P.; SALMON, W. C. (Ed.). Scientific explanation. Minneapolis: University of Minnesota Press, 1989. p. 410-499. (Minnesota studies in the philosophy of science, 13). Disponível em: <http://mcps.umn.edu/philosophy/VolumeXIII.htm>. Acesso em: 22 jan. 2018.

KNIGHT-BARDSLEY, A.; McNEILL, K. L. Teachers' pedagogical design capacity for scientific argumentation. Science Education, Hoboken, v. 100, n. 4, p. 645-672, 2016.

KUHN, D. Science as argument: implications for teaching and learning scientific thinking. Science Education, Hoboken, v. 77, n. 3, p. 319-337, 1993.

MARTIN, M. Concepts of science education: a philosophical analysis. London: Scott, Foresman, 1972.

MARTINS, I.; OGBORN, J.; KRESS, G. Explicando uma explicação. Ensaio: pesquisa em educação em ciências, Belo Horizonte, v. 1, n. 1, p. 1-14, 1999. Disponível em: < https://doi. org/10.1590/1983-21171999010104>. Acesso em: 22 jan. 2018.

MCNEILL, K. L. Elementary students' views of explanation, argumentation, and evidence, and their abilities to construct arguments over the school year. Journal of Research in Science Teaching, Hoboken, v. 48, n. 7, p. 793-823, 2011.

MCNEILL, K. L.; KRAJCIK, J. Scientific explanations: characterizing and evaluating the effects of teacher's instructional practices on student learning. Journal of Research in Science Teaching, Hoboken, v. 45, n. 1, p. 53-78, 2008.

MCNEILL, K. L.; LIZOTTE, D. J.; KRAJCIK J. Supporting students’ construction of scientific explanations by fading scaffolds in instructional materials. Journal of the Learning Sciences, Philadelphia, v. 15, n. 2, p. 153-191, 2006.

MILLAR, R.; OSBORNE, J. Beyond 2000: science education for the future. London: King's College London, School of Education, 1998.

MILNE, C. Philosophically correct science stories?: examining the implications of heroic science stories for school science. Journal of Research in Science Teaching, Hoboken, v. 35, n. 2, p. 175-187, 1998.

MORTIMER, E. F. Microgenetic analysis and the dynamic of explanations in science classroom. In: CONFERÊNCIA DE PESQUISA SÓCIO-CULTURAL, 3., 2000, Campinas. Anais... Campinas: Unicamp, 2000. Disponível em: <https://www.fe.unicamp.br/eventos/ br2000/ling2.htm>. Acesso em: 22 jan. 2018.

MORTIMER, E. F; SCOTT, P. Atividade discursiva nas salas de aula de ciências: uma ferramenta sociocultural para analisar e planejar o ensino. Investigações em Ensino de Ciências, Porto Alegre, v. 7, n. 3, p. 283-306, 2002. Disponível em: <https://www.if.ufrgs. br/cref/ojs/index.php/ienci/article/view/562/355>. Acesso em: 22 jan. 2018.

NAGEL, E. The structure of science: problems in the logic of scientific explanation. New York: Harcourt, Brace \& World, 1961. 
Explicações no ensino de ciências: revisando o conceito ...

NATIONAL RESEARCH COUNCIL. Taking science to school: learning and teaching science in grades K-8. Washington: National Academies Press, 2007.

NORRIS, S. P.; PHILLIPS, L. M. How literacy in its fundamental sense is central to scientific literacy. Science Education, Hoboken, v. 87, n. 2, p. 224-240, 2003.

NORRIS, S. P. et al. A theoretical framework for narrative explanation in science. Science Education, Hoboken, v. 89, n. 4, p. 535-563, 2005.

OGBORN, J. et al. Explaining science in the classroom. Buckingham: Open University Press, 1996.

OSBORNE, J. F.; ERDURAN, S.; SIMON, S. Enhancing the quality of argumentation in school science. Journal of Research in Science Teaching, Hoboken, v. 41, n. 10, p. 994-1020, 2004.

OSBORNE, J. F.; PATTERSON, A. Author's response to "for whom is argument and explanation a necessary distinction?: a response to Osborne and Patterson" by Berland and McNeill. Science Education, Hoboken, v. 96, n. 5, p. 814-817, 2012.

Scientific argument and explanation: a necessary distinction? Science Education, Hoboken, v. 95, n. 4, p. 627-638, 2011.

PEREIRA, A. P.; LIMA JUNIOR, P. R. M.; RODRIGUES, R. F. Explaining as mediated action: an analysis of pre-service teacher's account of forces of inertia in non-inertial frames of reference. Science \& Education, Dordrecht, v. 25, n. 3, p. 343-362, 2016.

RIBEIRO, R. M. L.; MARTINS, I. O potencial das narrativas como recurso para o ensino de ciências: uma análise em livros didáticos de física. Ciência \& Educação, Bauru, v. 13, n. 3, p. 293-309, 2007. Disponível em: <https://doi.org/10.1590/S1516-73132007000300002>. Acesso em: 22 jan. 2018.

SÁ, L. P.; KASSEBOEHMER, A. C.; QUEIROZ, S. L. Esquema de argumento de Toulmin como instrumento de ensino: explorando possibilidades. Ensaio: pesquisa em educação em ciências, Belo Horizonte, v. 16, n. 3, p. 147-170, 2014. Disponível em: < https://doi. org/10.1590/1983-21172014160307>. Acesso em: 22 jan. 2018.

SALMON, W. C. Four decades of scientific explanation. In: KITCHER, P.; SALMON, W. C. (Ed.). Scientific explanation. Minneapolis: University of Minnesota Press, 1989. p. 3-219. (Minnesota studies in the philosophy of science, 13). Disponível em: <http://mcps.umn. edu/philosophy/VolumeXIII.htm>. Acesso em: 22 jan. 2018.

Scientific explanation and the causal structure of the world. Princeton:

Princeton University Press, 1984.

SAMPSON, V.; CLARK, D. B. Assessment of the ways students generate arguments in science education: current perspectives and recommendations for future directions. Science Education, Hoboken, v. 92, n. 3, p. 447-472, 2008.

SANDOVAL, W. A. Conceptual and epistemic aspects of students' scientific explanations. The Journal of the Learning Sciences, Philadelphia, v. 12, n. 1, p. 5-51, 2003. 
Rodrigues, R. F.; Pereira, A. P.

SASSERON, L. H.; CARVALHO, A. M. P. Uma análise de referenciais teóricos sobre a estrutura do argumento para estudos de argumentação no ensino de ciências. Ensaio: pesquisa em educação em ciências, Belo Horizonte, v. 13, n. 3, p. 243-262, 2011. Disponível em: <http://dx.doi.org/10.1590/1983-21172011130315>. Acesso em: 22 jan. 2018.

SIMON, S.; ERDURAN, S.; OSBORNE, J. Learning to teach argumentation: research and development in the science classroom. International Journal of Science Education, Abingdon, v. 28, n. 2-3, p. 235-260, 2006.

SMITH, B. H. Narrative versions, narrative theories. In: MITCHELL, W. J. T. (Ed.). On narrative. Chicago: University of Chicago Press, 1981, p. 213-236.

THAGARD, P. Explanatory identities and conceptual change. Science \& Education, Dordrecht, v. 23, n. 7, p. 1531-1548, 2014.

TOULMIN, S. The uses of argument. Cambridge: Cambridge University Press, 1958.

WARD, P. D. The call of distant mammoths: why the ice age mammoths disappeared.

New York: Copernicus, 1997.

WERTSCH, J. V.; ROEDIGER, H. L. Collective memory: conceptual foundations and theoretical approaches. Memory, Abingdon, v. 16, n. 3, p. 318-326, 2008.

ZOHAR, A.; NEMET, F. Fostering students' knowledge and argumentation skills through dilemmas in human genetics. Journal of Research in Science Teaching, Hoboken, v. 39, n. 1, p. 35-62, 2002.

Artigo recebido em 16/03/2017. Aceito em 22/08/2017.

Endereço para contato: Avenida Bento Gonçalves, 9500, CEP:

91501-970, Porto Alegre, RS, Brasil. 